\title{
Tachyons, Lamb shifts and superluminal chaos
}

\author{
R. Tomaschitz ${ }^{\mathrm{a}}$ \\ Department of Physics, Hiroshima University, 1-3-1 Kagami-yama, Higashi-Hiroshima 739-8526, Japan
}

Received 13 August 1999 and Received in final form 7 February 2000

\begin{abstract}
An elementary account on the origins of cosmic chaos in an open and multiply connected universe is given; there is a finite region in the open 3-space in which the world-lines of galaxies are chaotic, and the mixing taking place in this chaotic nucleus of the universe provides a mechanism to create equidistribution. The galaxy background defines a distinguished frame of reference and a unique cosmic time order; in this context superluminal signal transfer is studied. Tachyons are described by a real Proca field with negative mass square, coupled to a current of subluminal matter. Estimates on tachyon mixing in the geometric optics limit are derived. The potential of a static point source in this field theory is a damped periodic function. We treat this tachyon potential as a perturbation of the Coulomb potential, and study its effects on energy levels in hydrogenic systems. By comparing the induced level shifts to high-precision Lamb shift measurements and QED calculations, we suggest a tachyon mass of $2.1 \mathrm{keV} / c^{2}$ and estimate the tachyonic coupling strength to subluminal matter. The impact of the tachyon field on ground state hyperfine transitions in hydrogen and muonium is investigated. Bounds on atomic transition rates effected by tachyon radiation as well as estimates on the spectral energy density of a possible cosmic tachyon background radiation are derived.
\end{abstract}

PACS. 05.45.Ac Low-dimensional chaos - 32.10.Fn Fine and hyperfine structure -98.70 .Vc Background radiations

\section{Introduction}

Our contemporary understanding of the global structure of the Universe is based on the assumption of a spacetime continuum, a curved 4-dimensional world. The observational evidence for this is actually scarce $[1,2]$, but it offers at any rate a very simple qualitative explanation of the cosmic redshifts. The second motivation to model the Universe as a Riemannian 4-manifold is the successful application of Riemannian geometry in explaining planetary perihelion shifts, and the deflection of light by gravitating bodies. These are local phenomena, which can be explained by introducing a Riemannian metric on a single coordinate chart. In cosmology, however, we are concerned with the global structure, with the topology of the 4-manifold.

In local problems of general relativity, Einstein's equations determine the evolution of the metric, once the energy-momentum tensor of the local gravitational sources is known. In cosmology, however, boundary conditions get crucial because we cannot assume asymptotic flatness. Einstein's equations certainly do not give any hint on the topology, and the evolution of the metric is likewise undetermined, because we do not really know the energymomentum tensor of the matter distribution in the Universe, nor the boundary conditions to be imposed. It is

\footnotetext{
a e-mail: roman@fusion.sci.hiroshima-u.ac.jp
}

in my opinion pointless to hunt for elusive laws of cosmic evolution, the modeling employed in such endeavors is always copied from finite classical or quantum systems, and there is no reason to assume that the Universe is a finite Hamiltonian system like any other. A much more promising approach to cosmology is to figure out possibilities of cosmic evolution, and to think over which local physical manifestations they can have.

The actual question is not so much what is the topology, but rather how does it evolve, because otherwise it is difficult to motivate why the cosmic 3 -space should have acquired, once and for all, a particular type of topology and metric. What we will advocate here is a cosmology with an open 3-space [3] that evolves by global deformations [4] in a way that the constant curvature of the 3space is retained. There are three conditions to be satisfied for the 3-space to admit a dynamical evolution by global deformations, and for space-time itself to provide a mechanism to generate chaos, which can account for the uniformity of the galaxy background. The 3-space must be open, multiply connected, and hyperbolic (of constant negative curvature) [5]. Hyperbolicity is needed to generate the instability of geodesics [6-8], and the infinite volume and a multiply connected topology are necessary to allow evolution by global metrical deformations; a pictorial description of such deformations can be found in [9]. Finally, the multiply connected topology confines certain unstable world-lines to a finite region, the center of the open 


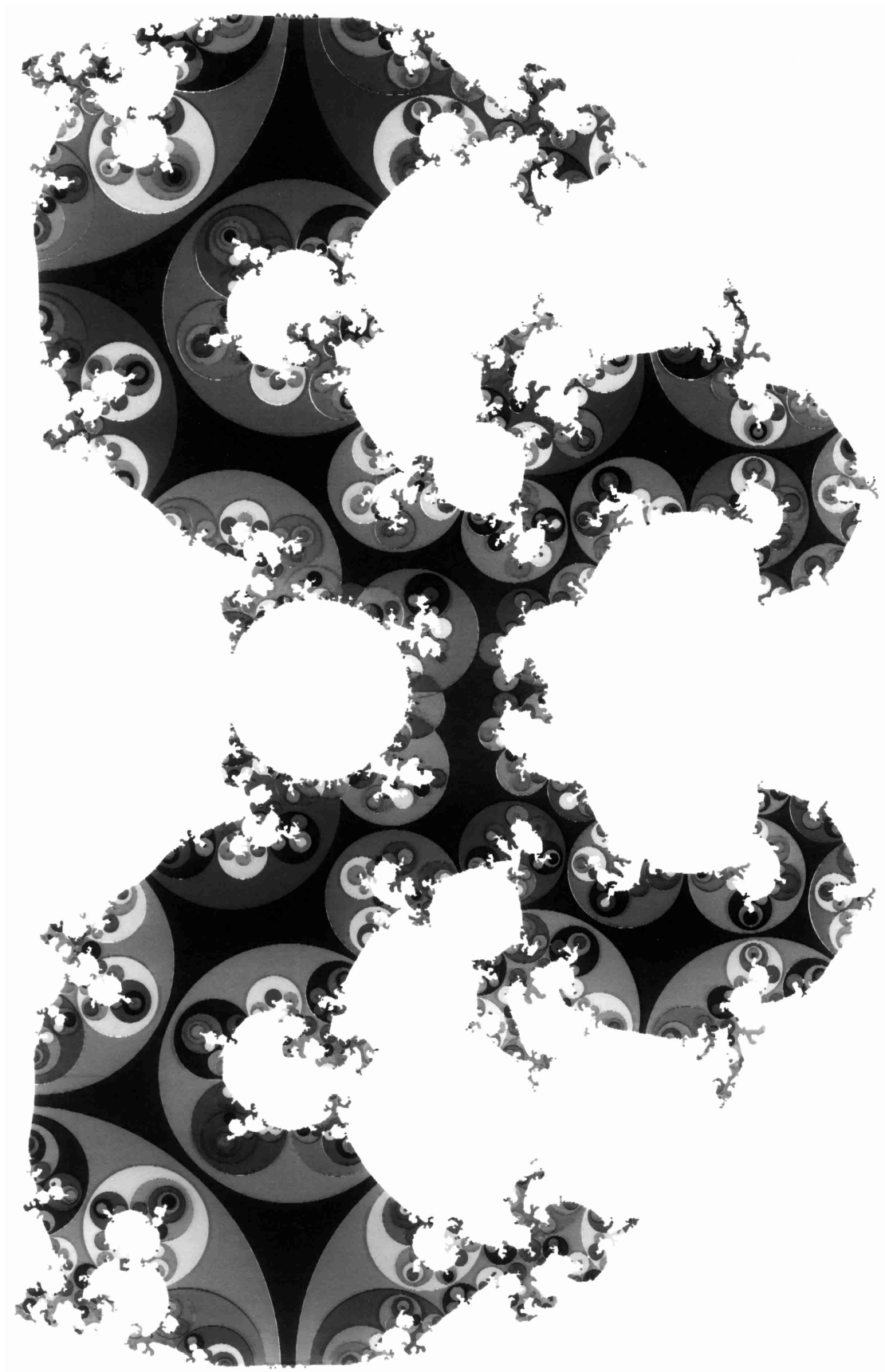

Fig. 1. Algorithmic construction of an open hyperbolic 3-manifold and the fractal limit set determining the chaotic trajectories. Depicted is a tiling in the complex plane. The tiles are defined by polygons composed of circular arcs. This tiling can be extended to three dimensions, into the upper half-space (Poincaré half-space $\mathrm{H}^{3}$, endowed with a hyperbolic metric) by placing hemispheres onto the circular arcs. In this way a tessellation of hyperbolic space is obtained, quite analogous to a crystal lattice in Euclidean space, though the lattice cells have infinite hyperbolic volume. The symmetry group of this lattice is a discrete subgroup of the Lorentz group. The lattice cells are polyhedra bounded by the complex plane and the hemispheres. The hyperbolic manifold is defined by identifying the polyhedral faces of a lattice cell in pairs with symmetry transformations of the lattice. (All cells are hyperbolically equivalent.) A geodesic in $\mathrm{H}^{3}$ is partitioned by the tessellation into arc pieces intersecting the individual cells of the lattice, and geodesic motion in the hyperbolic manifold (defined by a polyhedral cell with face identification) is realized by projecting these arcs into the manifold by means of the symmetry transformations. If the projected $\mathrm{H}^{3}$-geodesic has its initial and terminal point in the fractal limit set of the tiling, then it intersects infinitely many tiles of the hyperbolic lattice, and its projection is dense in a finite domain of the hyperbolic manifold, and has the Bernoulli property there. Chaotic trajectories are confined to this finite domain, the chaotic nucleus of the hyperbolic manifold. If the end points of the $\mathrm{H}^{3}$-geodesic are not quite in the limit set, but close to it, then its projection is still regular, but shadowed by chaotic trajectories [6]. (This figure is part of a deformation sequence discussed in [5], but was omitted there.) 
3 -space $[5,10,11]$, so that they can get chaotic there, $c f$. the caption of Figure 1. Chaoticity is an efficient mechanism to create equidistribution, but the actual problem is to explain the inhomogeneities in the galaxy distribution. The time evolution of the world-lines of galaxies depends on the expansion and on the global deformations that the 3 -space undergoes. Concepts like mixing and ergodicity do not reflect the time evolution, since they are based on the geometric shapes of geodesics of infinite length. The mixing in the center tends to create a uniform distribution, but inhomogeneities will always remain in a finite time [9]. The evolution of the global topological structure of the 3-space can get visible in the angular anisotropy of the temperature of the cosmic microwave background radiation [12]. Adiabatic global deformations of the 3-space lead to a distortion of the black-body spectrum, due to slightly angular dependent frequency shifts. These shifts can be absorbed in the Planck distribution by introducing an angular dependent temperature variable [13]. As mentioned, a non-trivial evolution of the hyperbolic 3space, via global deformations including topology changes, requires an open universe [14]. Unlike compact Riemann surfaces, hyperbolic 3-manifolds of finite volume are rigid, that is, they cannot be deformed without distorting the constant curvature. The spectral properties of Riemann surfaces of infinite area, the two-dimensional analog to the cosmic 3-space, are discussed in a very transparent way in [15]; also see Poincaré's collected papers on this subject [16].

On a microscopic level, a multiply connected structure of space-time was suggested by Weyl already in the twenties [4], and this idea has been revived and extended by El Naschie and Nottale in terms of a fractal microdimensionality of space-time [17-19]. Parity violation, as a microscopic topological self-interference phenomenon, was considered in [20]. Mixmaster cosmologies, chaos in the Einstein equations, and its various ramifications are discussed by Rugh in [21,22]. Examples of relativistic chaos in local gravitational fields, including an invariant characterization of unstable orbits, can be found in [23,24].

In this paper, we study superluminal signals (tachyons [25-32]); the comoving galaxy frame defines a distinguished cosmic time order, which unambiguously defines the causality of superluminal signal transfer [33,34], $c f$. Section 5 for more discussion on that. Tachyons are regarded as the eigenmodes of a real vector field with negative mass square. Like the electromagnetic field, the tachyon field is conformally coupled to the background geometry, so that the frequencies of the spectral elementary waves scale inversely proportional to the curvature radius of the cosmic 3 -space. This allows, despite the time variation of the background geometry, to use the thermodynamic equilibrium formalism when dealing with the cosmic tachyon background radiation, and to scale the time dependence of the eigenmodes into the temperature variable, which becomes in this way a function of cosmic time.

In Section 2, we sketch the classical mechanics of tachyons in a Robertson-Walker cosmology, the energy concept for tachyons in the comoving galaxy frame, the wave equation for tachyons, a Proca equation with negative mass square, and its coupling to a subluminal current. Tachyons are introduced as an extension of the photon concept, as transversally propagating particles with negative mass square. This suggests a coupling mechanism to subluminal matter in analogy to electrodynamics. The first part of this section is a concise summary of research published in $[35,36]$, to keep this paper self-contained. The second part is devoted to new investigations, to quantitative estimates concerning the mixing of tachyonic rays in the geometric optics limit, which is in fact much more pronounced than for galaxies; its efficiency can be estimated if one knows the tachyon mass, derived in Section 3, the expansion factor, and some other cosmological parameters. We calculate the potential of a static point source in this field theory, as well as the vector potential of a stationary current of subluminal particles carrying tachyonic charge, and introduce tachyonic $E$ and $B$ fields and the tachyonic analog to Maxwell's equations.

In Section 3, we treat this tachyon potential as a perturbation of the Coulomb potential in the Schrödinger equation, and estimate its effect on bound states in hydrogen-like systems. By comparing the induced level shifts to the discrepancy between Lamb shift measurements and QED calculations in hydrogen, we calculate the tachyon mass, $m_{\mathrm{t}} \approx m_{\mathrm{e}} / 238 \approx 2.15 \mathrm{keV} / c^{2}$, and the tachyonic fine structure constant, $\beta \approx 1.0 \times 10^{-13} \approx$ $0.66 \alpha^{6}$, which are the two parameters determining the tachyon potential. With these numbers, we calculate the tachyonic $B$-field of a nucleus of spin one-half, and estimate the effect of this field and of the perturbed ground state wave function on the ground state hyperfine intervals of muonium and hydrogen.

In Section 4, we calculate bounds on atomic absorption and emission rates for tachyon radiation, and give estimates on the speed of tachyons emitted in atomic transitions. The spectral energy density of the cosmic tachyon background radiation is studied. The tachyonic energy density deviates from the Rayleigh-Jeans low frequency limit, and we conclude, by comparing tachyonic and photonic absorption rates, that this deviation could be observable for wavelengths in the meter range. In the conclusion, Section 5, we continue our discussion of tachyons in this cosmological setting, in particular with regard to causality and second quantization. In the Appendix, the tachyonic correction to the ground state wave function of a hydrogenic system is calculated.

\section{The wave equation for tachyons, tachyon mixing and tachyon potentials}

We consider tachyons in an open Robertson-Walker cosmology with hyperbolic 3-space, endowed with the line element $\mathrm{d} \lambda^{2}=-\mathrm{d} \tau^{2}+a^{2}(\tau) \mathrm{d} \sigma^{2}$ (comoving coordinates). We use as coordinate representation of the 3 -space the Poincaré half-space $\mathrm{H}^{3}$, defined by the metric $\mathrm{d} \sigma^{2}=$ $u^{-2}\left(\mathrm{~d} u^{2}+|\mathrm{d} \xi|^{2}\right)$, with Cartesian coordinates $u, \xi_{1}, \xi_{2}$; $u>0$, and $\xi=\xi_{1}+\mathrm{i} \xi_{2} \cdot \mathrm{d} \sigma^{2}$ induces constant negative 
curvature -1 on this half-space [37]. Tachyonic world-lines are defined by a Hamilton-Jacobi equation with negative mass square, $g^{\mu \nu} S_{, \mu} S_{, \nu}=\mu^{2}\left(\mu^{2}>0\right.$ in our notation, with $g_{\mu \nu}$ defined by $\left.\mathrm{d} \lambda^{2}\right)$. The tachyon mass $\mu$ varies in cosmic time inversely proportional to the curvature radius of the 3 -space, $\mu=m_{\mathrm{t}} / a(\tau)$. (The wave equation for tachyons is conformally coupled, see the discussion following (2.3), which requires a tachyon mass varying in cosmic time.) This cosmic time scaling reminds of the varying fundamental constants of Eddington, Milne, and Dirac [38-41]. It is sufficient to determine the world-lines along the $u$ semiaxis of $\mathrm{H}^{3}$, all other trajectories can be obtained by means of symmetry transformations [37], since $\mathrm{H}^{3}$ is homogeneous. The tachyonic world-lines read

$$
u(\tau)=\exp \left(\delta(s) \int a^{-1} \mathrm{~d} \tau\right), \quad \delta(s):=\frac{s}{\sqrt{s^{2}-m_{\mathrm{t}}^{2}}},
$$

with an integration parameter $|s|>m_{\mathrm{t}}$. If we focus on trajectories orthogonal to the complex plane, we can use the action $S=-\sqrt{s^{2}-m_{\mathrm{t}}^{2}} \int a^{-1} \mathrm{~d} \tau+s \log u$, the trajectories (2.1) being recovered from $\partial S / \partial s=$ const. Tachyonic energy, velocity and momentum read as

$$
\begin{aligned}
E & =\frac{\mu}{\sqrt{|\mathbf{v}|^{2}-1}}=\frac{1}{a(\tau)} \sqrt{s^{2}-m_{\mathrm{t}}^{2}} \\
|\mathbf{v}| & =|\delta(s)|, \quad|\mathbf{p}|=\frac{|s|}{a(\tau)}
\end{aligned}
$$

In the case of photons, the eikonal approximation is exact in Robertson-Walker cosmologies, because the electromagnetic potential is conformally coupled to the background metric, which means here that the frequencies scale with the inverse of the expansion factor. We also want to retain this property for particles with negative mass square. We view tachyons as an extension of the photon concept, and use as wave equation a Proca equation with negative mass square,

$$
\frac{1}{\sqrt{-g}} \frac{\partial\left(\sqrt{-g} F^{\alpha \beta}\right)}{\partial x^{\beta}}-\mu^{2} A^{\alpha}=j^{\alpha},
$$

for a real vector potential $A_{\alpha}$ with $F_{\alpha \beta}=A_{\beta, \alpha}-A_{\alpha, \beta}$ and $\mu=m_{\mathrm{t}} / a(\tau), m_{\mathrm{t}}>0$. The choice of this wave equation as an extension of electrodynamics rather than of subluminal classical mechanics is also suggested by the fact that tachyons do not have a rest mass. The current in (2.3) is supposed to be structured like in electrodynamics, and thus a well defined interaction mechanism of a tachyon field with subluminal particles, analogous to classical electrodynamics, is specified by (2.3). Tachyonic world-lines are analogous to the light-rays of geometric optics; tachyons do not carry any kind of charge; electric and tachyonic charge is a property of subluminal particles, the sources of tachyonic and electromagnetic fields.

The curvature radius of the 3 -space is $R a(\tau), a\left(\tau_{0}\right)=1$ at the present epoch. To restore the natural units, we replace in the wave equation $m_{\mathrm{t}}$ by $m_{\mathrm{t}} c / \hbar$. The phase of the spectral modes of freely propagating waves coincides with the classical action. (This is easy to check for plane waves transversally propagating along the $u$ semiaxis, whose phase is the action specified after (2.1); the spectral decomposition of the wave equation is derived in [35].) Hence, energy and momentum are proportional to frequency and wave vector, so that $\omega=E / \hbar$, and $\lambda=\hbar /|\mathbf{p}|$; the eikonal approximation is exact, due to the conformal coupling of the wave field, achieved by the time variation of the tachyon mass. Likewise, group and particle velocity coincide, $\left|\mathbf{v}_{\text {gr }}\right|=c^{2}\left|\mathbf{v}_{\text {phase }}\right|^{-1}=c|\delta(s)|$, and can be made arbitrarily large by the choice of the integration parameter (spectral variable) $s$.

Next we turn to tachyon mixing in an open and multiply connected 3 -space, $c f$. Section 1 . At first we consider a tachyon moving along the $u$-semi-axis of $\mathrm{H}^{3}$ with speed $c \delta(s)$, passing through $\left(\tau_{0}, u_{0}=R\right)$. We consider a Milne universe, so that $a(\tau)=\tau / \tau_{0}, \tau_{0}=1 / H_{0} \approx 14.4 \mathrm{Gyr}$ (based on $h_{0} \approx 0.68,[42]$ ), and $R_{0}=c / H_{0} \approx 1.3 \times 10^{28} \mathrm{~cm}$. Equation (2.1) now reads as $u=R\left(\tau / \tau_{0}\right)^{\delta}$. We assume $\delta>0$, so that the tachyon moves the $u$-semiaxis upwards, having been emitted at $\left(\tau_{\mathrm{em}}, u_{\mathrm{em}}\right), \tau_{\mathrm{em}}<\tau_{0}$. At the present epoch $\tau_{0}$, the metric distance between $u_{0}$ and $u_{\mathrm{em}}$ is $d\left(u_{0}, u_{\mathrm{em}}\right)=R \log \left(u_{0} / u_{\mathrm{em}}\right)$. If this distance is $n$-times the curvature radius, then $u_{\mathrm{em}} / R=\mathrm{e}^{-n}$, and $\Delta \tau_{n}:=\tau_{0}-\tau_{\mathrm{em}}=\tau_{0}(1-\exp (-n / \delta))$. This relation holds for every tachyonic world-line, because of the homogeneity of $\mathrm{H}^{3}$. If $n / \delta \approx 1$, we find $\Delta \tau_{n} \approx 0.6 \tau_{0}$. The frequencies of the tachyonic spectral waves relate to the velocity of the classical tachyonic world-lines via $h \nu=m_{\mathrm{t}} c^{2}\left(\mathbf{v}^{2} / c^{2}-1\right)^{-1 / 2}, \mathbf{v}^{2} / c^{2}=\delta^{2}$, at the present epoch, as pointed out above. We find $\delta \approx 3.6 \times 10^{8}$, with a tachyon mass of $2.15 \mathrm{keV} / c^{2}$ (see after (3.12)), if the tachyon is emitted in a hydrogen ground state hyperfine transition (see after (4.7)). The tachyon is trapped in the chaotic nucleus of the 3 -space ( $c f$. the caption of Fig. 1 and $[5,10,11]$ for details), if its covering geodesic in $\mathrm{H}^{3}$ has its initial and terminal points in the limit set of the hyperbolic tiling. If the diameter of the chaotic nucleus of the 3 -space is of the order of the curvature radius, and if the tachyon was emitted at $\tau_{\mathrm{em}} \approx 0.4 \tau_{0}$, then the trajectory consists of roughly $n \approx \delta$ arcs in the lattice cell representing the hyperbolic 3 -space, $c f$. Figure 1 , because its covering geodesic in $\mathrm{H}^{3}$ intersected $n$ tiles within the past $0.6 \tau_{0}$ years. Even for moderate $n$, these arcs are very uniformly distributed in the center of the 3-space, by virtue of the Bernoulli property. Indeed, computer experiments on compact Riemann surfaces [8] demonstrate impressively, that a very uniform cover of the manifold is already achieved with rather few arc pieces. Whatever the speed of the tachyon, its world-line gets dense in the chaotic nucleus in the limits $\tau \rightarrow 0, \infty$, provided the initial or terminal point of the covering trajectory lies in the limit set, so that it intersects infinitely many lattice cells. If the expansion factor has turning or inflection points, a tachyonic worldline can even get dense in the chaotic nucleus within a finite time [9]. Another startling feature of tachyons, caused by the space expansion but independent of the topology of the 3-space, are mirror images; a tachyon may appear 
simultaneously at different locations in the geodesic rest frame of a uniformly moving observer [35].

The finite chaotic nucleus is not a differentiable manifold, but the entropy of the geodesic flow trapped in this domain can be defined very much like on a compact manifold. The topological and measure theoretic definitions of entropy turn out to be equivalent; these equilibrium entropies coincide with the Hausdorff dimension of the limit set of the covering group [7]. Both entropy definitions assume that the covering trajectories extend from infinity to infinity, reaching in both limits $\tau \rightarrow 0, \infty$ the boundary of the covering space. This happens to be the case in the Milne universe, but other expansion factors may result in a truncation of the covering trajectories in one or both of these limits (appearance of horizons), and hence in truncated Bernoulli sequences [14], and then neither the topological nor the measure theoretic entropy concept is applicable. One should also keep in mind that these entropies are static; they relate to the geometric shape of the trajectories (which is in fact independent of the expansion factor, apart from a possible truncation), but are otherwise not affected by the time evolution of the geodesic flow. The efficiency of the mixing mechanism within a finite period of cosmic time crucially depends both on the time evolution of the trajectories and the Hausdorff dimension of the limit set.

Next we turn to the generation of tachyonic waves and to the tachyon potential of a static point source. We use locally geodesic coordinates, i.e., replace the space-time metric by Minkowski space, $\eta_{\alpha \beta}:=\operatorname{diag}\left(-c^{2}, 1,1,1\right)$, and restore the natural units. The field equations (2.3) decompose into four components, $\left(\square+\mu^{2}\right) A_{\alpha}=-c^{-1} j_{\alpha}$, with the d'Alembertian $\square:=\eta^{\mu \nu} \partial_{\mu} \partial_{\nu}$ and $\mu:=m_{\mathrm{t}} c / \hbar>0$, subject to the Lorentz condition $A^{\alpha}{ }_{, \alpha}=0$. We write $j^{\alpha}=(\rho, \mathbf{j})$, and calculate the potential of a static point source defined by $\rho=q \delta(\mathbf{x})$ and $\mathbf{j}=0$, where $q$ is the tachyonic charge of the source,

$$
A^{0}=\frac{q}{4 \pi c} \frac{1}{r}(\cos (\mu r)+\tilde{\lambda} \sin (\mu r)), \quad \mathbf{A}=0 .
$$

Contrary to massless or subluminal fields, we cannot determine the integration constant $\tilde{\lambda}$ in (2.4) by a decay condition at infinity. To demonstrate that $\tilde{\lambda}=0$, we write the wave field as

$$
\begin{aligned}
& A_{\alpha}(x)=\int_{\mathrm{R}^{4}} G\left(x-x^{\prime}\right) j_{\alpha}\left(x^{\prime}\right) \mathrm{d} x^{\prime}, \\
& \left(\square+\mu^{2}\right) G(t, \mathbf{x})=-c^{-1} \delta(t) \delta(\mathbf{x}) .
\end{aligned}
$$

For positive mass square, $\mu^{2}<0$ in our notation, the general solution of this inversion problem is standard, for negative mass square, it can readily be inferred from the subluminal case by analytic continuation in the mass parameter, $m \rightarrow \pm \mathrm{i} m_{\mathrm{t}}$. (Some care must be taken with regard to the branch cuts of the Bessel functions.) Tachyons can never reach subluminal speed, i.e., they cannot connect events with timelike separation, and therefore the boundary condition for the Green function is to vanish in the interior of the light cone. This already determines $G$ uniquely, without any further decay conditions, as

$$
\begin{aligned}
G(t, \mathbf{x})=\frac{1}{4 \pi} \delta & \left(r^{2}-c^{2} t^{2}\right) \\
& -\frac{\mu}{8 \pi} \theta\left(r^{2}-c^{2} t^{2}\right) \frac{J_{1}\left(\mu \sqrt{r^{2}-c^{2} t^{2}}\right)}{\sqrt{r^{2}-c^{2} t^{2}}} .
\end{aligned}
$$

This Green function is time symmetric, there is no tachyonic retarded or advanced Green function supported outside the light cone; $G$ in (2.7) is the analogue to $(1 / 2)\left(G_{\text {ret }}+G_{\text {adv }}\right)$. (Retarded and advanced Green functions of subluminal particles are supported on the two disconnected components of the interior light cone.) A discussion of tachyonic Liénard-Wiechert potentials, in particular a detailed study of time-symmetric wave propagation via the Green function (2.7), its semiclassical meaning, its cosmological causality interpretation, and its relation to the cosmic absorber theory of WheelerFeynman [43] will be given elsewhere. We readily calculate, via (2.7) and (2.5), the potential of a static tachyonic charge; the spatial integration is trivial, and the time integral over the Bessel function is standard, and we recover (2.4) with $\tilde{\lambda}=0$. In Section 3, we will study frequency shifts induced by this potential in hydrogen, and infer in this way the tachyon mass and the coupling constant of the tachyon potential.

In analogy to Maxwell's equations, one can introduce tachyonic $E$ and $B$ fields related to the vector potential $A_{\alpha}$ by

$$
\begin{aligned}
E_{i} & =c^{-1} F_{i 0} \quad, \quad F_{i j}=\varepsilon_{i j k} B^{k}, \\
B^{k} & =(1 / 2) \varepsilon^{k i j} F_{i j}=\varepsilon^{k i j} A_{j, i}=\operatorname{rot} \mathbf{A},
\end{aligned}
$$

so that the field equations (2.3) read (in Minkowski space) as

$$
\begin{aligned}
& \operatorname{div} \mathbf{B}=0 \quad, \quad \operatorname{rot} \mathbf{E}+c^{-1} \partial \mathbf{B} / \partial \tau=0, \\
& \operatorname{div} \mathbf{E}=\rho-c^{-1} \mu^{2} A_{0}, \quad \operatorname{rot} \mathbf{B}-c^{-1} \partial \mathbf{E} / \partial \tau=c^{-1} \mathbf{j}+\mu^{2} \mathbf{A} .
\end{aligned}
$$

Evidently, the vector potential is a measurable quantity, as there is no gauge freedom. Tachyonic $E$ and $B$ fields act on subluminal particles (mass $m$ ) via a Lorentz force, based on the Hamilton-Jacobi equation $\eta^{\mu \nu}\left(S_{, \mu}-c^{-1} q A_{\mu}\right)\left(S_{, \nu}-\right.$ $\left.c^{-1} q A_{\nu}\right)=-m^{2} c^{2}$.

In the next section, we will need the tachyonic vector potential generated by a stationary current, which can easily be calculated via (2.5) and (2.7),

$$
A_{\alpha}(\mathbf{x})=\frac{1}{4 \pi c} \int_{\mathrm{R}^{3}} \frac{\cos \left(\mu\left|\mathbf{x}-\mathbf{x}^{\prime}\right|\right)}{\left|\mathbf{x}-\mathbf{x}^{\prime}\right|} j_{\alpha}\left(\mathbf{x}^{\prime}\right) \mathrm{d} \mathbf{x}^{\prime} .
$$

The $B$-field corresponding to this potential reads as

$$
\mathbf{B}(\mathbf{x})=\frac{1}{4 \pi c} \int_{\mathrm{R}^{3}} \operatorname{grad}_{\mathbf{x}}\left(\frac{\cos \left(\mu\left|\mathbf{x}-\mathbf{x}^{\prime}\right|\right)}{\left|\mathbf{x}-\mathbf{x}^{\prime}\right|}\right) \times \mathbf{j}\left(\mathbf{x}^{\prime}\right) \mathrm{d} \mathbf{x}^{\prime},
$$

which is the Biot-Savart law for negative mass square. The following procedures are familiar from electrodynamics 
with a finite photon mass, $c f$. [44] and references therein. The gradient expansion of the spatial part of the vector potential (2.10) reads

$$
\begin{aligned}
4 \pi c \mathbf{A}=r^{-1} & \cos (\mu r) \int \mathbf{j}\left(\mathbf{x}^{\prime}\right) \mathrm{d} \mathbf{x}^{\prime} \\
& -\int\left(\nabla\left(r^{-1} \cos (\mu r)\right) \cdot \mathbf{x}^{\prime}\right) \mathbf{j}\left(\mathbf{x}^{\prime}\right) \mathrm{d} \mathbf{x}^{\prime}+\ldots
\end{aligned}
$$

This expansion only holds if $\mu\left|\mathbf{x}^{\prime}\right| \ll 1$ on the support of the current. This is an important restriction, in addition to the usual assumption that the coordinate origin is centered at the support of the current, and $r$ is large compared to the diameter of the support. In Section 3 we will derive $\mu^{-1}=\lambda_{t}^{\mathrm{C}} \approx 0.9 \AA$, which disqualifies the gradient expansion for macroscopic charge distributions (also compare the discussion of $(2.16,2.17))$, but for nuclear dipole moments it is quite appropriate. and

In discrete notation, $\mathbf{j}\left(\mathbf{x}^{\prime}\right)=\sum q \mathbf{v}^{\prime}=(\mathrm{d} / \mathrm{d} t) \sum q \mathbf{x}^{\prime}$,

$$
\begin{aligned}
2 \sum q\left(\mathbf{x} \cdot \mathbf{x}^{\prime}\right) \mathbf{v}^{\prime}=\sum q\left(\left(\mathbf{x} \cdot \mathbf{x}^{\prime}\right) \mathbf{v}^{\prime}\right. & \left.-\left(\mathbf{x} \cdot \mathbf{v}^{\prime}\right) \mathbf{x}^{\prime}\right) \\
& +(\mathrm{d} / \mathrm{d} t) \sum q\left(\mathbf{x} \cdot \mathbf{x}^{\prime}\right) \mathbf{x}^{\prime}
\end{aligned}
$$

the time derivative of $\sum q\left(\mathbf{x} \cdot \mathbf{x}^{\prime}\right) \mathbf{x}^{\prime}$ vanishes, because the current is stationary. Hence, by a standard vector identity, we may write $(2.12)$ as

$$
\begin{aligned}
4 \pi c \mathbf{A}= & (V(r) / 2) \int \mathbf{x} \times\left(\mathbf{x}^{\prime} \times \mathbf{j}\left(\mathbf{x}^{\prime}\right)\right) \mathrm{d} \mathbf{x}^{\prime}, \\
& \nabla\left(r^{-1} \cos (\mu r)\right)=V(r) \mathbf{x} \\
V(r):= & -r^{-3} \cos (\mu r)(1+\mu r \tan (\mu r)),
\end{aligned}
$$

or, with the magnetic dipole moment,

$$
\begin{aligned}
& \mathbf{A}=(4 \pi)^{-1} \nabla\left(r^{-1} \cos (\mu r)\right) \times \mathbf{m} \\
& \mathbf{m}:=(2 c)^{-1} \int \mathbf{x} \times \mathbf{j}(\mathbf{x}) \mathrm{d} \mathbf{x} .
\end{aligned}
$$

The $B$-field is then obtained, via (2.8) and a further vector identity, as

$$
\begin{aligned}
\mathbf{B}=(4 \pi)^{-1}(\mathbf{m} \cdot \nabla) \nabla\left(r^{-1} \cos (\mu r)\right) \\
-(4 \pi)^{-1} \mathbf{m} \operatorname{div}\left(\nabla\left(r^{-1} \cos (\mu r)\right)\right),
\end{aligned}
$$

or, by means of $\operatorname{div}\left(\nabla\left(r^{-1} \cos (\mu r)\right)\right)=-\mu^{2} r^{-1} \cos (\mu r)$, as

$$
\begin{aligned}
\mathbf{B}=\frac{1}{4 \pi} \frac{\cos (\mu r)}{r^{3}} & {\left[\left(1+\mu r \tan (\mu r)-\frac{1}{3} \mu^{2} r^{2}\right)\right.} \\
\times & \left.\left(3\left(\mathbf{m} \cdot \frac{\mathbf{x}}{r}\right) \frac{\mathbf{x}}{r}-\mathbf{m}\right)+\frac{2}{3} \mu^{2} r^{2} \mathbf{m}\right] .
\end{aligned}
$$

The first term in (2.15) is formally a multiple of the electromagnetic dipole field, but the second term mimics an external magnetic field (anti-)parallel to the dipole. A similar (but non-periodic) external field emerges in electrodynamics with a finite photon mass, and the dipole field of the Earth has been used to obtain bounds on this mass [44]. However, the expansion underlying (2.13-2.15) only applies to microscopic dipoles; in the next section we will study the effect of a nuclear tachyonic $B$-field on hyperfine transitions.

The zeros of the tachyon potential are spaced in intervals $\Delta r=\pi \lambda_{\mathrm{t}}^{\mathrm{C}} \approx 2.9 \AA$. Hence, if the tachyon potential is generated by an extended macroscopic charge distribution, it will not be so easily detectable, because of averaging effects, and the same holds for tachyonic $E$ and $B$ fields. For example, anomalous magnetic moments determined by $g-2$ experiments would not be affected in a noticeable way, as the $B$-field is generated by a macroscopic magnet. We consider a tachyonic charge $q$, uniformly distributed in a ball of radius $R$. The tachyon potential of this charge is the real part of

$$
\begin{aligned}
\varphi_{R}(\mathbf{x}) & =\frac{q}{4 \pi c}\left(\frac{4 \pi}{3} R^{3}\right)^{-1} \int_{\left|\mathbf{x}^{\prime}\right| \leq R} \frac{\mathrm{e}^{\mathrm{i} \mu\left|\mathbf{x}-\mathbf{x}^{\prime}\right|}}{\left|\mathbf{x}-\mathbf{x}^{\prime}\right|} \mathrm{d} \mathbf{x}^{\prime} \\
& =\frac{3}{8 \pi c} \frac{q}{R^{3}} \frac{\mathrm{i}}{\mu r} \int_{0}^{R} r^{\prime} \mathrm{d} r^{\prime}\left(\mathrm{e}^{\mathrm{i} \mu\left|r-r^{\prime}\right|}-\mathrm{e}^{\mathrm{i} \mu\left(r+r^{\prime}\right)}\right) .
\end{aligned}
$$

For $|\mathbf{x}|>R$, we find

$$
\begin{aligned}
\varphi_{R}(r) & =-\frac{3}{4 \pi c} \frac{q}{\mu^{2} R^{2}}\left(\cos (\mu R)-\frac{1}{\mu R} \sin (\mu R)\right) \frac{\mathrm{e}^{\mathrm{i} \mu r}}{r} \\
& =\frac{q}{4 \pi c}\left(1+O\left(\mu^{2} R^{2}\right)\right) \frac{\mathrm{e}^{\mathrm{i} \mu r}}{r} .
\end{aligned}
$$

Clearly, if $\mu R \gg 1$, this effectively means a reduction of the charge, but in the case of nuclei, the opposite limit, as indicated in (2.17), safely applies.

\section{The effect of the tachyon potential on Lamb shifts and hyperfine transitions in hydrogenic systems}

We treat the local Euclidean limit of the tachyon potential (2.4) (with $\tilde{\lambda}=0$ ) as a perturbation of the Coulomb potential, and write the Schrödinger equation for a hydrogen-like system as

$$
\Delta \Psi+\frac{2 m}{\hbar^{2}}(E-U) \Psi=0, \quad U(r)=-\frac{\alpha}{r}+\frac{\beta}{r} \cos (\mu r),
$$

$\alpha>0, \mu=m_{\mathrm{t}} c / \hbar>0$. We will investigate bound states of (3.1), and calculate level shifts induced by the tachyon potential. By assuming that these shifts can account for the discrepancy of experimental and theoretical Lamb shifts in hydrogen, we derive the tachyon mass and the coupling constant $\beta$ of the tachyon potential. We will 
focus on the ground state and the $2 S$ Lamb shifts in hydrogen, because there are several recent and rather independent high precision measurements available, and the present QED calculations for hydrogen are more reliable than for any other hydrogen-like system.

In (3.1) we use the standard ansatz for a spherically symmetric potential, $\Psi=r^{-1} u_{l, E}(r) Y_{l, m}(\vartheta, \varphi)$, with spherical harmonics normalized on the unit sphere, $Y_{0,0}=$ $(4 \pi)^{-1 / 2}$, etc. The radial equation reads

$$
\begin{aligned}
\frac{\mathrm{d}^{2}}{\mathrm{~d} r^{2}} u_{l, E}(r)+\frac{2}{a}\left(\frac{E}{\alpha}+\frac{1}{r}\right. & -\frac{a}{2} \frac{l(l+1)}{r^{2}} \\
& \left.-\lambda \frac{\cos (\mu r)}{r}\right) u_{l, E}(r)=0,
\end{aligned}
$$

with Bohr radius $a=\hbar^{2} /(m \alpha)$ and expansion parameter $\lambda:=\beta / \alpha$. Standard perturbation theory gives

$$
\begin{aligned}
E & =E_{0}+\lambda E_{1}+O\left(\lambda^{2}\right) \\
E_{1} & =\int_{0}^{\infty} u_{0}(r) \overline{u_{0}(r)} \frac{\alpha}{r} \cos (\mu r) \mathrm{d} r
\end{aligned}
$$

with the eigenvalues $E_{0}=-\alpha /\left(2 a n^{2}\right)$ and the normalized radial eigenfunctions $u_{0}$ of the unperturbed Coulomb problem,

$$
\left(-\frac{\mathrm{d}^{2}}{\mathrm{~d} r^{2}}+\frac{1}{a^{2} n^{2}}-\frac{2}{a r}+\frac{l(l+1)}{r^{2}}\right) u_{0}(r)=0,
$$

$\int_{0}^{\infty} u_{0} \overline{u_{0}} \mathrm{~d} r=1$. We will focus on the $1 S_{1 / 2}, 2 S_{1 / 2}$ and $2 P_{1 / 2,3 / 2}$ levels; the corresponding solutions of (3.4) and the corrections (3.3) to the Coulomb levels $E_{0}(n)$ read

$$
\begin{aligned}
u_{0}^{(n=1, l=0)} & =\frac{2}{a^{3 / 2}} r \mathrm{e}^{-r / a} \\
E_{1}^{(n=1, l=0)} & =\frac{\alpha}{a} \frac{1-a^{2} \mu^{2} / 4}{\left(1+a^{2} \mu^{2} / 4\right)^{2}} \\
u_{0}^{(n=2, l=0)} & =\frac{r \mathrm{e}^{-r /(2 a)}}{\sqrt{2} a^{3 / 2}}\left(1-\frac{r}{2 a}\right) \\
E_{1}^{(n=2, l=0)} & =\frac{\alpha}{4 a} \frac{\left(1-6 a^{2} \mu^{2}+a^{4} \mu^{4}\right)\left(1-2 a^{2} \mu^{2}\right)}{\left(1+a^{2} \mu^{2}\right)^{4}}, \\
u_{0}^{(n=2, l=1)} & =\frac{r^{2} \mathrm{e}^{-r /(2 a)}}{2 \sqrt{2} \sqrt{3} a^{5 / 2}}, \\
E_{1}^{(n=2, l=1)} & =\frac{\alpha}{4 a} \frac{\left(1-6 a^{2} \mu^{2}+a^{4} \mu^{4}\right)}{\left(1+a^{2} \mu^{2}\right)^{4}} .
\end{aligned}
$$

It is convenient to define the energy levels as positive, so that $E(1 S)=-\left(E_{0}^{n=1}+E_{1 S}^{\mathrm{rel}, \mathrm{QED}}\right)+E_{1 S}^{\mathrm{tach}}, E_{1 S}^{\mathrm{tach}}:=$ $-\lambda E_{1}^{(n=1, l=0)}$, and analogously, $E_{2 S}^{\text {tach }}:=-\lambda E_{1}^{(n=2, l=0)}$ and $E_{2 P}^{\mathrm{tach}}:=-\lambda E_{1}^{(n=2, l=1)}$. The standard theoretical correction to $E_{0}$ is indicated by $E^{\text {rel,QED }}$, comprising the relativistic correction as well as the tabulated Lamb shift (including finite-size effects, etc.). Furthermore, we define

$$
\hat{\alpha}:=\alpha /(\hbar c), \quad \hat{\beta}:=\beta /(\hbar c), \quad \hat{\mu}:=a \mu,
$$

so that $a=\hbar /(m c \hat{\alpha})$. Clearly, $\hat{\beta}$ is the tachyonic analogue to the electric fine structure constant $\hat{\alpha} \approx 1 / 137$. Quite analogously to $\alpha=e^{2} /(4 \pi)$, we may write $\beta=$ $q_{\mathrm{e}} q_{\mathrm{p}} /(4 \pi)$, with the tachyonic charges of electron and proton, respectively, and $\hat{\mu} \hat{\alpha}$ denotes the tachyon mass in units of the reduced mass of electron and proton, $m \approx$ $m_{\mathrm{e}} \approx 0.511 \mathrm{MeV} / c^{2}$. We write $E_{1 S-2 S}^{\mathrm{tach}}:=E_{1 S}^{\mathrm{tach}}-E_{2 S}^{\mathrm{tach}}$, and $E_{1 S-2 S}^{\mathrm{th}}:=E_{1 S}^{\mathrm{th}}-E_{2 S}^{\mathrm{th}}$, with the theoretical standard results $E_{1 S}^{\mathrm{th}}:=-\left(E_{0}^{n=1}+E_{1 S}^{\mathrm{rel}, \mathrm{QED}}\right)$ and $E_{2 S}^{\mathrm{th}}:=$ $-\left(E_{0}^{n=2, l=0}+E_{2 S}^{\text {rel,QED }}\right)$, and analogously for the other levels. The experimental $1 S-2 S$ transition energy is denoted by $E_{1 S-2 S}^{\text {ex }}$. The difference between the measured energy and the theoretical standard result is denoted by $E_{1 S-2 S}^{\mathrm{ex}-\mathrm{th}}:=E_{1 S-2 S}^{\mathrm{ex}}-E_{1 S-2 S}^{\mathrm{th}}$, and the tachyonic correction $E_{1 S-2 S}^{\text {tach }}$ is supposed to compensate this difference. We find, by means of (3.5-3.7),

$$
\begin{aligned}
& \frac{E_{2 S-2 P}^{\mathrm{tach}}}{m c^{2}}=\frac{\hat{\alpha} \hat{\beta}}{2} \frac{y\left(1-6 y+y^{2}\right)}{(1+y)^{4}}, y:=\hat{\mu}^{2} \\
& \frac{E_{1 S-2 S}^{\mathrm{tach}}}{m c^{2}}=\frac{\hat{\alpha} \hat{\beta}}{4} \frac{14 y^{5}-3 y^{4}-96 y^{3}-175 y^{2}-360 y-48}{(4+y)^{2}(1+y)^{4}}
\end{aligned}
$$

$$
\frac{E_{1 S-2 P}^{\mathrm{tach}}}{m c^{2}}=\frac{\hat{\alpha} \hat{\beta}}{4} \frac{16 y^{5}+y^{4}-158 y^{3}-351 y^{2}-328 y-48}{(4+y)^{2}(1+y)^{4}} .
$$

Sign changes are determined by the positive zeros of $E^{\operatorname{tach}}(\hat{\mu})$; in $(3.9)$ we find $\hat{\mu}_{(1)} \approx 2.414$ and $\hat{\mu}_{(2)} \approx 0.414$, the zero of $(3.10)$ is $\hat{\mu} \approx 1.903$, and the zero of $(3.11)$ is $\hat{\mu} \approx 2.011$.

A recent measurement [45] of the ground state and the $2 S$ Lamb shifts in hydrogen gives $L_{1 S}^{\mathrm{ex}}(\mathrm{H})=$ $8172.876(29) \mathrm{MHz}$ and $L_{2 S}^{\mathrm{ex}}(\mathrm{H})=1045.0079(72) \mathrm{MHz}$, respectively. As for the standard theory, the current estimates are $L_{1 S}^{\mathrm{th}}(\mathrm{H})=8172.797(40) \mathrm{MHz}$ and $L_{2 S}^{\mathrm{th}}(\mathrm{H})=$ $1045.0036(50) \mathrm{MHz},[45,46]$. We have $E_{1 S-2 S}^{\mathrm{ex}-\mathrm{th}}=L_{1 S}^{\mathrm{th}}-$ $L_{1 S}^{\mathrm{ex}}+L_{2 S}^{\mathrm{ex}}-L_{2 S}^{\mathrm{th}}$, and in the experimental work the theoretical $2 \mathrm{P}$ Lamb shifts $[47,48]$ are used, so that we can assume $L_{2 P}^{\mathrm{ex}}=L_{2 P}^{\mathrm{th}}$ in $E_{2 S-2 P}^{\mathrm{ex}-\mathrm{th}}=L_{2 S}^{\mathrm{th}}-L_{2 S}^{\mathrm{ex}}+L_{2 P}^{\mathrm{ex}}-L_{2 P}^{\mathrm{th}}$. Accordingly, $\nu_{1 S-2 S}^{\text {ex }- \text { th }}=-0.0747(810) \mathrm{MHz}$, and $\nu_{2 S-2 P}^{\text {ex }- \text { th }}=$ $-0.0043(122) \mathrm{MHz}$. The tachyon mass can then be determined from the quotient of (3.10) and (3.9) $\left(y:=\hat{\mu}^{2}\right)$,

$$
\frac{\nu_{1 S-2 S}^{\mathrm{ex}-\mathrm{th}}}{\nu_{2 S-2 P}^{\mathrm{ex}-\mathrm{th}}}=\frac{1}{2 y}\left(1-2 y-\frac{16(4-y)(1+y)^{4}}{\left(1-6 y+y^{2}\right)(4+y)^{2}}\right) \text {. }
$$

This equation has at least one and at most three positive solutions, depending on the frequency quotient. With the indicated frequencies, we find two solutions, $\hat{\mu}=0.576$, and $\hat{\mu}_{2}=2.576$, and the corresponding tachyonic coupling constants then follow from (3.9) or (3.10): $\hat{\beta}=$ $1.0 \times 10^{-13} \approx 0.66 \hat{\alpha}^{6}$, and $\hat{\beta}_{2}=-9.3 \times 10^{-13}$. A recent Lamb shift measurement in singly-ionized helium suggests that $\hat{\mu}$ and $\hat{\beta}$ is the relevant solution, see after (3.13). Tachyon mass and Compton wave length then read as $m_{\mathrm{t}}=m \hat{\alpha} \hat{\mu} \approx 2.15 \mathrm{keV} / c^{2}$ and $\lambda_{\mathrm{t}}^{\mathrm{C}}=\mu^{-1} \approx 238 \lambda_{\mathrm{e}}^{\mathrm{C}} \approx$ 
$0.92 \AA$, respectively. For comparison, a tachyon mass of $3.0 \mathrm{keV}$ and a tachyonic coupling constant of $6.4 \times 10^{-14}$ was obtained in [36] by Bohr quantization and a sinpotential, with slightly different input data for $\nu_{2 S-2 P}^{\mathrm{ex}-\mathrm{th}}(\mathrm{H})$ and $\nu_{1 S-2 S}^{\text {ex }- \text { th }}(\mathrm{H})$. Other promising candidates for a determination of the tachyon potential in hydrogen are the very precisely measured $2 S-6 S$ and $2 S-6 D_{5 / 2}$ intervals, cf. [49], or combinations $\left(2 S-4 P_{1 / 2,3 / 2}\right)-(1 / 4)(1 S-2 S)$, cf. [50], or $\left(2 S-4 S, 4 D_{5 / 2}\right)-(1 / 4)(1 S-2 S)$, cf. [51]. However, if higher levels are involved, the calculations get tedious due to the increasing order of the Laguerre polynomials in the eigenfunctions. The recently measured muonium $1 S-2 S$ interval, $\nu_{1 S-2 S}^{\text {ex }- \text { th }}(\mathrm{M})=5.6(9.8) \mathrm{MHz}$, cf. [52], is by two orders too inaccurate. As $\hat{\beta}$ is positive, the tachyonic charges of proton and electron are equal in sign, and we assume, that they are equal in magnitude as well, so that $q_{\mathrm{e}}=q_{\mathrm{p}}=: q$, and $q^{2} / e^{2}=1.4 \times 10^{-11}$. Evidence for this assumption could be provided by Lamb shifts in positronium [53], but current measurements and QED calculations do not match the precision of the hydrogen results.

The energy differences (3.9-3.11) also apply to hydrogenic ions, if we perform the substitutions

$$
\hat{\alpha} \rightarrow \hat{\alpha} Z, \quad \hat{\beta} \rightarrow \hat{\beta} Z, \quad \hat{\mu} \rightarrow \hat{\mu} / Z
$$

The tachyon mass $m_{\mathrm{t}}$ should be independent of the charge number of the nucleus, which requires the indicated scaling of $\hat{\mu}$. The linear $Z$-scaling of the tachyonic fine structure constant is based on the assumption that the potentials of tachyonic charges are additive like electric ones. $m \approx m_{\mathrm{e}}$ is now the reduced mass of nucleus and electron. In [35] we assumed a power law, $\hat{\beta} \rightarrow \hat{\beta} Z^{7}$, to account for the rather large discrepancies between the QED calculations and the Lamb shift measurements in highly charged hydrogenic ions, but the error bounds in these measurements are still very large, see below.

The tachyonic correction $\Delta E_{L}^{\text {tach }}:=$ $\left(m c^{2}\right)^{-1} E_{2 S-2 P_{1 / 2}}^{\mathrm{tach}}, \quad c f . \quad(3.9)$, is to be compared to $\Delta E_{L}:=\left(m c^{2}\right)^{-1} E_{2 S-2 P_{1 / 2}}^{\mathrm{ex}-\mathrm{th}}$, where $E_{2 S-2 P_{1 / 2}}^{\mathrm{ex}-\mathrm{th}}=L^{\mathrm{th}}-L^{\mathrm{ex}}$, in terms of the $2 S-2 P$ shift. The best result currently available for hydrogenic ions is that for singly-ionized helium, $\Delta E_{L}\left(\mathrm{He}^{+}\right)=3.2(24.3) \times 10^{-16}$, calculated with $L^{\mathrm{ex}}\left(\mathrm{He}^{+}\right)=14041.13(17) \mathrm{MHz}$ and $L^{\text {th }}\left(\mathrm{He}^{+}\right)=14041.17(13) \mathrm{MHz}$, cf. [54]. The tachyonic correction, $\Delta E_{L}^{\mathrm{tach}}\left(\mathrm{He}^{+}\right)=4.5 \times 10^{-17}$, is still by one order of magnitude smaller than the present discrepancy between experiment and theoretical standard result. Had we taken the roots $\hat{\mu}_{2}$ and $\hat{\beta}_{2}$ for tachyon mass and tachyonic fine structure constant, as given after (3.12), we would have arrived at a shift $\Delta E_{L}^{\mathrm{tach}}\left(\mathrm{He}^{+}, \hat{\mu}_{2}, \hat{\beta}_{2}\right)=2.8 \times 10^{-15}$, which is only narrowly within the error bounds defined by $L^{\mathrm{ex}, \mathrm{th}}\left(\mathrm{He}^{+}\right)$.
For medium-sized or heavy ions, we may consider the $Z \rightarrow \infty$ limit of (3.9) and (3.11),

$$
\begin{aligned}
\Delta E_{L}^{\mathrm{tach}}= & \frac{E_{2 S-2 P}^{\mathrm{tach}}}{m c^{2}} \sim \frac{\hat{\alpha} \hat{\beta}}{2} \hat{\mu}^{2} \approx 1.2 \times 10^{-16}, \\
& \frac{E_{1 S-2 P}^{\mathrm{tach}}}{m c^{2}} \sim-\frac{3}{4} \hat{\alpha} \hat{\beta} Z^{2} \approx-5.5 \times 10^{-16} Z^{2} .
\end{aligned}
$$

The following examples are taken from Table 20 of [55]; as conversion constant we use $h /\left(m c^{2}\right) \approx 8.09 \times$ $10^{-21}$ s. In the low $Z$-range: $\Delta E_{L}\left(\mathrm{~F}^{8+}\right)=3.3(29.0) \times$ $10^{-11}, \Delta E_{L}\left(\mathrm{O}^{7+}\right)=3.2(9.7) \times 10^{-11}$. In the intermediate $Z$-range: $\Delta E_{L}\left(\mathrm{Ar}^{17+}\right)=2.9(3.2) \times 10^{-9}$, $\Delta E_{L}\left(\mathrm{~S}^{15+}\right)=0.88(61) \times 10^{-9}$. As for ground state Lamb shifts, we define $\Delta E_{1 S 2 P}:=\left(m c^{2}\right)^{-1} E_{1 S-2 P_{1 / 2,3 / 2}}^{\mathrm{ex}-\text { th }}$ (to be compared to $\left(m c^{2}\right)^{-1} E_{1 S-2 P}^{\mathrm{tach}}$ in $\left.(3.14)\right)$, put

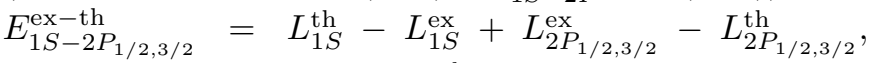
and assume $L_{2 P_{1 / 2,3 / 2}}^{\mathrm{ex}}=L_{2 P_{1 / 2,3 / 2}}^{\mathrm{th}}$, as done in experimental work. We find in the intermediate $Z$-range, cf. Table 19 of [55], $\Delta E_{1 S 2 P}\left(\mathrm{Ar}^{17+}\right)=-9.8(33.0) \times$ $10^{-9}$ and $\Delta E_{1 S 2 P}\left(\mathrm{Kr}^{35+}\right)=-1.9(10.0) \times 10^{-7}$, and for heavy ions, $\Delta E_{1 S 2 P}\left(\mathrm{Au}^{78+}\right)=-1.2(30.0) \times 10^{-5}$, and $\Delta E_{1 S 2 P}\left(\mathrm{U}^{91+}\right)=-6.7(26.0) \times 10^{-6}$. It is clear that these results are too inaccurate to allow for a test of the asymptotic estimates (3.14).

Next we study the effect of the tachyon field on the hyperfine splitting in hydrogen and muonium. The formalism is more or less the same as in the derivation of the Fermi formula, $c f$. [53] and references therein. We start with the Pauli equation for a subluminal particle of mass $m_{\mathrm{e}}$, coupled by minimal substitution to the tachyon potential,

$$
\frac{1}{\mathrm{i}} \nabla_{0} \Psi=\frac{\hbar}{2 m_{\mathrm{e}}}\left(\nabla_{k} \nabla^{k}+\frac{q}{\hbar c} \sigma_{i} \varepsilon^{i k l} A_{l, k}\right) \Psi
$$

$\nabla_{\alpha}:=\partial_{\alpha}-\mathrm{i} q /(\hbar c) A_{\alpha}$, with tachyonic charge density $\rho=$ $q \Psi^{\mathrm{t}^{*}} \Psi$. The Hamilton operator corresponding to (3.15) reads as

$$
H=-\frac{\hbar^{2}}{2 m_{\mathrm{e}}}\left(\nabla_{k} \nabla^{k}+\frac{q}{\hbar c} \boldsymbol{\sigma} \cdot \operatorname{rot} \mathbf{A}\right)-\frac{q}{c} A_{0} .
$$

The perturbation generated by the tachyonic $B$-field is accordingly

$$
H_{\mathrm{HFS}}=-q_{\mathrm{e}} /\left(m_{\mathrm{e}} c\right) \mathbf{S} \cdot \mathbf{B}, \quad \mathbf{S}=(\hbar / 2) \boldsymbol{\sigma},
$$

with $\mathbf{A}$ and $\mathbf{B}$ as in (2.13-2.15). Here, $m_{\mathrm{e}}$ and $q_{\mathrm{e}}$ denote mass and tachyonic charge of the electron, $\mathbf{S}$ is the spin operator of the electron, and $\boldsymbol{\sigma}$ the vector of Pauli matrices. We will apply $H_{\text {HFS }}$ only to wave fields with radial symmetry, when calculating expectation values. Hence, when integrating over the unit sphere, we may replace in the B-field $(\mathbf{m} \cdot \mathbf{x}) \mathbf{x}$ by $(1 / 3) \mathbf{m} r^{2}$ without changing the integral. Therefore we can replace in the first term of (2.14) $(\mathbf{m} \cdot \nabla) \nabla$ by $(1 / 3) \mathbf{m} \Delta$, and use in the subsequent 
calculations

$$
\begin{aligned}
\mathbf{B} & =-\frac{1}{6 \pi} \mathbf{m} \Delta \frac{\cos (\mu r)}{r}, \\
\Delta \frac{\cos (\mu r)}{r} & =-4 \pi \delta(\mathbf{x})-\mu^{2} \frac{\cos (\mu r)}{r} .
\end{aligned}
$$

We consider a nucleus of spin one-half, so that

$$
\mathbf{m}=g_{\mathrm{n}} \mu_{\mathrm{n}} \mathbf{I} / \hbar, \quad \mu_{\mathrm{n}}=\frac{q_{\mathrm{n}} \hbar}{2 m_{\mathrm{n}} c}, \quad \mathbf{I}=(\hbar / 2) \boldsymbol{\sigma} .
$$

I is the nuclear spin operator, $\mu_{\mathrm{n}}$ denotes the tachyonic nuclear magneton (not to be confused with the tachyon mass $\left.\mu=m_{\mathrm{t}} c / \hbar\right), m_{\mathrm{n}}$ is the nuclear mass, $q_{\mathrm{n}}$ is the tachyonic charge of the nucleus, and the nuclear $g$-factor is denoted by $g_{\mathrm{n}}$. In the case of muonium, we take the Dirac value $g_{\mathrm{n}}=2$; when calculating the very tiny effect of the tachyon potential, we neglect of course QED corrections. For the same reason, we have already put the $g$-factor of the electron equal two in (3.16), according to the non-relativistic limit (3.15) of the Dirac equation.

We calculate the expectation value of $H_{\mathrm{HFS}}$ with the normalized ground state wave function of the unperturbed Coulomb problem, which reads $\Psi(\mathbf{x})=\pi^{-1 / 2} a^{-3 / 2} \mathrm{e}^{-r / a}$, with Bohr radius $a=\hbar /(m c \hat{\alpha})$, where $m$ denotes the reduced mass of electron and nucleus. We do not consider hydrogen-like ions here, the nucleus carries one positive electric charge and spin $1 / 2$. The spin space of nucleus and electron is generated by a basis $|F, M\rangle$, defined in the usual way by the $z$-component of $\mathbf{F}:=\mathbf{S}+\mathbf{I}$, $\mathbf{F}_{3}|F, M\rangle=M \hbar|F, M\rangle$ and $\mathbf{F}^{2}|F, M\rangle=F(F+1) \hbar^{2}|F, M\rangle$. (Both $\mathbf{S}$ and $\mathbf{I}$ are spin one-half operators.) We so obtain a singlet $|0,0\rangle$ and a triplet $|1,0\rangle,|1, \pm 1\rangle$, as base vectors. As $2 \mathbf{S} \cdot \mathbf{I}=\mathbf{F}^{2}-\mathbf{I}^{2}-\mathbf{S}^{2}$ and $\mathbf{I}^{2}=\mathbf{S}^{2}=(3 / 4) \hbar^{2}$, we find

$$
\langle\mathbf{S} \cdot \mathbf{I}\rangle_{F}:=\langle F, M|\mathbf{S} \cdot \mathbf{I}| F, M\rangle=\left(\hbar^{2} / 2\right)(F(F+1)-3 / 2) ;
$$

$F$ can only take the values one and zero, and this expectation value is independent of $M$. The unperturbed ground state wave function is thus $|\Psi, F, M\rangle=\Psi(\mathbf{x})|F, M\rangle$, and we calculate with $(3.17-3.19)$,

$$
\begin{gathered}
\left\langle\Psi, F, M\left|H_{\mathrm{HFS}}\right| \Psi, F, M\right\rangle=-\frac{2}{3} \frac{q_{\mathrm{e}} g_{\mathrm{n}} \mu_{\mathrm{n}}}{m_{\mathrm{e}} c \hbar}\langle\mathbf{S} \cdot \mathbf{I}\rangle_{F}|\Psi(0)|^{2} \\
\times\left(1+\frac{\mu^{2}}{4 \pi|\Psi(0)|^{2}} \int|\Psi(\mathbf{x})|^{2} \frac{\cos (\mu r)}{r} \mathrm{~d} \mathbf{x}\right) \\
\left\langle H_{\mathrm{HFS}}\right\rangle_{F}=\frac{4}{3} g_{\mathrm{n}} \hat{\alpha}^{4} \frac{\langle\mathbf{S} \cdot \mathbf{I}\rangle_{F}}{\hbar^{2}} m_{\mathrm{e}} c^{2} \frac{m_{\mathrm{e}}}{m_{\mathrm{n}}}\left(1+\frac{m_{\mathrm{e}}}{m_{\mathrm{n}}}\right)^{-3} \\
\times \frac{\hat{\beta}}{\hat{\alpha}}\left(\frac{\kappa(1-\kappa)}{(1+\kappa)^{2}}-1\right) \\
\hat{\beta}:=\frac{q_{\mathrm{e}} q_{\mathrm{n}}}{4 \pi \hbar c}, \quad \kappa:=\frac{1}{4 \hat{\alpha}^{2}} \frac{m_{\mathrm{t}}^{2}}{m_{\mathrm{e}}^{2}}\left(1+\frac{m_{\mathrm{e}}}{m_{\mathrm{n}}}\right)^{2}
\end{gathered}
$$

If we drop the last two factors in (3.22), and put $g_{\mathrm{n}}=2$, we recover Fermi's formula for the ground state hyperfine splitting. We have $\langle\mathbf{S} \cdot \mathbf{I}\rangle_{F=1}=\hbar^{2} / 4$ and $\langle\mathbf{S} \cdot \mathbf{I}\rangle_{F=0}=$ $-3 \hbar^{2} / 4$, cf. (3.20). The tachyonic $B$-field of the nucleus changes the theoretical standard result (Fermi formula with QED corrections, etc., $c f$. [53]) for the ground state hyperfine interval by an amount $\Delta E^{\text {tach }}(B)=$ $\left\langle H_{\mathrm{HFS}}\right\rangle_{F=1}-\left\langle H_{\mathrm{HFS}}\right\rangle_{F=0}$, inducing a frequency shift

$$
\begin{aligned}
\Delta \nu^{\operatorname{tach}}(B) & =\Delta \nu^{\mathrm{F}} \frac{\hat{\beta}}{\hat{\alpha}}\left(\frac{\kappa(1-\kappa)}{(1+\kappa)^{2}}-1\right), \\
\Delta \nu^{\mathrm{F}} & :=\frac{4}{3} g_{\mathrm{n}} \hat{\alpha}^{4} \frac{m_{\mathrm{e}} c^{2}}{h} \frac{m_{\mathrm{e}}}{m_{\mathrm{n}}}\left(1+\frac{m_{\mathrm{e}}}{m_{\mathrm{n}}}\right)^{-3} .
\end{aligned}
$$

We find $\kappa=0.083$, based on $m_{\mathrm{t}}=2.15 \mathrm{keV} / c^{2}, m_{\mathrm{e}} / m_{\mathrm{t}} \approx$ 238 and $m_{\mathrm{n}}=\infty$. Moreover, $\hat{\beta} / \hat{\alpha}=1.4 \times 10^{-11}$, see after (3.12).

In (3.21-3.25), we used the unperturbed ground state wave function of the Coulomb problem, neglecting the $\lambda$-term in $(3.2)$, because $H_{\mathrm{HFS}}=O(\lambda)$. (We defined $\lambda=\hat{\beta} / \hat{\alpha}, c f .(3.2,3.8)$.) Nevertheless, there is still an $O(\lambda)$-contribution of the perturbed ground state wave function (3.1) to the hyperfine splitting. The electromagnetic hyperfine splitting as given by the Fermi formula reads

$$
\left\langle H_{\mathrm{HFS}}\right\rangle_{\mathrm{EM}}=\frac{1}{3} \frac{e^{2} g_{\mathrm{n}}}{m_{\mathrm{n}} m_{\mathrm{e}} c^{2}}\langle\mathbf{S} \cdot \mathbf{I}\rangle_{F}|\Psi(0)|^{2},
$$

which follows from (3.21) by substituting $q_{\mathrm{e}}=e, q_{\mathrm{n}}=-e$, and $\mu=0$. In (3.26) we have to use for $|\Psi(0)|^{2}$ the perturbed ground state function as defined by (3.1) and calculated to order $O(\lambda)$ in the Appendix. This perturbation results in a shift

$$
\Delta \nu^{\operatorname{tach}}(\Psi)=\left.\Delta \nu^{\mathrm{F}} \frac{\hat{\beta}}{\hat{\alpha}} a^{3 / 2} \frac{\hat{u}_{1}(r)}{r}\right|_{r=0},
$$

cf. (A.12, A.9). In (A.9) we insert $\hat{\mu}=0.576$ (see after $(3.12))$, so that $a^{3 / 2}\left(\hat{u}_{1}(r) / r\right)_{r=0} \approx-3.35$. Hence, the total frequency shift induced by the tachyon field of the nucleus (singly charged, spin one-half) is

$$
\Delta \nu^{\mathrm{tach}}:=\Delta \nu^{\mathrm{tach}}(B)+\Delta \nu^{\mathrm{tach}}(\Psi) \approx-6.0 \times 10^{-11} \Delta \nu^{\mathrm{F}},
$$

to be added to the theoretical standard result, denoted in the following by $\Delta \nu^{\mathrm{QED}}$.

In muonium, the Fermi formula gives $\Delta \nu^{\mathrm{F}}(\mathrm{M})=4453839.4(5) \mathrm{kHz} \quad$ (with $g_{\mathrm{n}}=2$, cf. [53]), so that $\Delta \nu^{\text {tach }}(\mathrm{M}) \approx-0.27 \mathrm{~Hz}$, which is too small to account for the current difference [56] of $\Delta \nu^{\mathrm{QED}}(\mathrm{M})=4463302563(510) \mathrm{Hz}$ and $\Delta \nu^{\mathrm{ex}}(\mathrm{M})=4463302765(53) \mathrm{Hz}$. In hydrogen, we have $\Delta \nu^{\mathrm{F}}(\mathrm{H})=1418.84029(14) \mathrm{MHz}$ (with $g_{\mathrm{n}}=5.58 \ldots$ ), so that $\Delta \nu^{\text {tach }}(\mathrm{H}) \approx-0.085 \mathrm{~Hz}$, which is to be compared to $\Delta \nu^{\mathrm{QED}}(\mathrm{H})=1420.45199(14) \mathrm{MHz}$ and $\Delta \nu^{\mathrm{ex}}(\mathrm{H})=1420.4057517667(9) \mathrm{MHz}, \quad c f$. [53]. In muonium, the contribution of the tachyon potential is 
negligible given the error bound on experimental and theoretical results; the same holds for the tachyonic correction to the muonium ground state Lamb shift, as mentioned after (3.12). As for hydrogen, the tachyonic correction is within the precision of the measured value, but the theoretical standard result is way too inaccurate, due to the poorly known charge distribution in a proton and other finite size effects.

\section{Cosmic tachyon background radiation}

As the tachyon frequencies depend on cosmic time only via a scale factor, $\nu=\nu_{0} / a(\tau), c f .(2.2)$ and the remarks following (2.3) and (4.7), one can use the equilibrium distribution of a free Bose gas to describe the cosmic tachyon background radiation and scale the time dependence of the frequencies into the temperature variable. Based on the free wave equation (2.3), one obtains by standard box quantization the spectral energy density

$$
\begin{aligned}
& \rho_{\text {tach }}(\nu) \mathrm{d} \nu=\frac{8 \pi}{h^{3}} \frac{E(p) p^{2} \mathrm{~d} p}{\exp (\beta E(p))-1} \\
& =\frac{8 \pi h}{c^{3}} \frac{\mathrm{d} \nu \nu^{2} \sqrt{\nu^{2}+m_{\mathrm{t}}^{2} c^{4} / h^{2}}}{\exp (\beta h \nu)-1}, \\
& E(p)=h \nu=c \sqrt{p^{2}-\left(m_{\mathrm{t}} c\right)^{2}}, \quad \beta^{-1}=k T(\tau), \\
& T(\tau)=T_{0} / a(\tau) .
\end{aligned}
$$

The general formalism and the conditions on wave lengths and curvature radius for Euclidean box quantization to apply are discussed in [35]. In the thermodynamic limit, the connectivity of the open 3-space does not affect the spectral energy density [13], apart from possible angular fluctuations in the temperature, generated by global metrical deformations, $c f$. Section 1 , and $\rho_{\text {tach }}(\nu)$ is even independent of the sign of the space curvature.

The tachyon mass of $m_{\mathrm{t}} \approx 2.15 \mathrm{keV} / c^{2}$ corresponds to a Compton wave length $\lambda_{\mathrm{t}}^{\mathrm{C}} \approx 0.9 \AA$, see after (3.12). This is the maximal wave length that can be attained, in the limit of infinite speed and zero energy, since $\lambda_{\mathrm{t}}=$ $\lambda_{\mathrm{t}}^{\mathrm{C}}\left(1+\left(\lambda_{\mathrm{t}}^{\mathrm{C}} \nu / c\right)^{2}\right)^{-1 / 2}$. As the tachyon radiation is in equilibrium with the photon background at $2.73 \mathrm{~K}$, we have $m_{\mathrm{t}} c^{2} /\left(k T_{0}\right) \approx 9.1 \times 10^{6}$ and $\beta h=1.76 \times 10^{-11} \mathrm{~s}$. $(\beta$ should not be confused with the coupling constant of the tachyon potential, $c f .(3.1)$; only the tachyonic fine structure constant $\hat{\beta}$ as defined in (3.8) is used in this section). In the high frequency limit, Wien's radiation law is still recovered, but not so the Rayleigh-Jeans law in the low frequency regime, because

$$
\rho_{\text {tach }}(\nu \rightarrow 0) \sim \frac{8 \pi}{c h} m_{\mathrm{t}} \nu k T,
$$

linear in frequency. Wien's displacement law is not valid either, as the peak of $\rho_{\operatorname{tach}}(\nu)$ depends on the tachyon mass. Defining $x:=\beta h \nu$ and $\gamma:=m_{\mathrm{t}} c^{2} /(k T)$, we find the location of the peak by solving

$$
\frac{x}{1-\mathrm{e}^{-x}}=2+\frac{x^{2}}{x^{2}+\gamma^{2}} \text {. }
$$

For the photon background, this means $x(\gamma=0) \approx 2.822$, and for the tachyon background $x(\gamma \rightarrow \infty) \approx 1.594$ applies, since, at the present epoch, $\gamma \approx 9.1 \times 10^{6}$. Hence, the tachyonic energy density is peaked in the microwave range, at $\nu_{\mathrm{t}}^{\text {peak }} \approx 90.6 \mathrm{GHz}\left(0.33 \mathrm{~cm}\right.$ or $\left.3.7 \times 10^{-4} \mathrm{eV}\right)$, rather close to the peak of the photon density at $\nu_{\mathrm{ph}}^{\text {peak }} \approx$ $160 \mathrm{GHz},\left(0.19 \mathrm{~cm}\right.$ or $\left.6.6 \times 10^{-4} \mathrm{eV}\right)$.

Next we turn to atoms in equilibrium with a mixture of photon and tachyon black-body radiation, and study the ratio of photon and tachyon transition rates [36]. The derivation of tachyonic transition rates is quite analogous to photonic ones, by standard equilibrium arguments not repeated here, but we sketch how this ratio relates to the Einstein coefficients and the photonic and tachyonic spectral energy densities. The quotient of the dipole matrix elements of electric and tachyonic charge and the absorption coefficients read

$$
\frac{\left|D_{m n}^{\mathrm{q}}\right|^{2}}{\left|D_{m n}^{\mathrm{e}}\right|^{2}}=\frac{\hat{\beta}}{\hat{\alpha}}, \quad B_{m n}^{\mathrm{e}, \mathrm{q}}=\frac{16}{3} \frac{\pi^{4}}{h^{2}}\left|D_{m n}^{\mathrm{e}, \mathrm{q}}\right|^{2} .
$$

The coefficients for spontaneous emission connect to the $B$-coefficients as

$$
A_{m n}^{\mathrm{e}}=4 \frac{h \nu^{3}}{c^{3}} B_{m n}^{\mathrm{e}}, \quad A_{m n}^{\mathrm{q}}=4 \frac{h \nu^{2}}{c^{3}} \sqrt{\nu^{2}+m_{\mathrm{t}}^{2} c^{4} / h^{2}} B_{m n}^{\mathrm{q}}
$$

where $\nu$ denotes the transition frequency. The spectral energy densities of photon and tachyon radiation relate as

$$
\rho_{\mathrm{tach}}(\nu)=\frac{\sqrt{\nu^{2}+m_{\mathrm{t}}^{2} c^{4} / h^{2}}}{\nu} \rho_{\mathrm{ph}}(\nu)
$$

The ratio of tachyon and photon transition rates is the same for absorptive, stimulated, and spontaneous transitions,

$$
\frac{R_{m n}^{\mathrm{tach}}}{R_{m n}^{\mathrm{ph}}} \leq \frac{B_{m n}^{\mathrm{q}}}{B_{m n}^{\mathrm{e}}} \frac{\rho_{\mathrm{tach}}(\nu)}{\rho_{\mathrm{ph}}(\nu)}=\frac{A_{m n}^{\mathrm{q}}}{A_{m n}^{\mathrm{e}}}=\frac{\hat{\beta}}{\hat{\alpha}} \frac{\sqrt{(h \nu)^{2}+m_{\mathrm{t}}^{2} c^{4}}}{h \nu}
$$

The dipole approximation is valid for radiation of wavelengths large compared to the size of the atom, so that the wave can be regarded as spatially constant. This is not the case for tachyon radiation, see after (4.1), and averaging effects lead to a reduction of $R^{\text {tach }}$, so that the estimate (4.7) for $R^{\mathrm{tach}} / R^{\mathrm{ph}}$ is only an upper bound. We find $R^{\mathrm{tach}} / R^{\mathrm{ph}}<3.0 \times 10^{-9}$ for the Ly- $\alpha$ lines of hydrogen $(10.2 \mathrm{eV})$, based on $m_{\mathrm{t}} \approx 2.15 \mathrm{keV} / c^{2}$ and $\hat{\beta} / \hat{\alpha} \approx 1.4 \times 10^{-11}$. In heavy ions, it is even more unlikely that atomic transitions are effected by tachyon radiation; $R^{\text {tach }} / R^{\text {ph }}<1.4 \times 10^{-11}$ for the Ly- $\alpha_{1}$ transition $(0.23 \mathrm{MeV})$ in hydrogenic uranium.

We give some estimates on the speed of tachyons emitted in atomic transitions. Tachyonic frequency and velocity relate via $h \nu=m_{\mathrm{t}} c^{2} a^{-1}(\tau)\left(\mathbf{v}^{2} / c^{2}-1\right)^{-1 / 2}$, because the classical action appears as the phase of the spectral waves, see (2.2) and after (2.3). As the tachyon velocity is (an integration) constant, we recover the same redshift scaling 
as for photons. (The tachyon mass of $2.15 \mathrm{keV}$ refers of course to the present epoch, $a\left(\tau_{0}\right)=1$.) If a tachyon is generated by a $\operatorname{Ly}-\alpha$ transition in a heavy hydrogenic ion, then its velocity is rather close to the speed of light. As for hydrogen transitions, we may use $\mathbf{v} \sim 2.15 \mathrm{keV} c /(h \nu)$, so that $\mathbf{v}($ Ly- $\alpha) \sim 210 c$; a $3 p_{3 / 2}-4 d_{5 / 2}$ transition effected by tachyon radiation results in $\mathbf{v}(0.66 \mathrm{eV}) \sim 3.2 \times 10^{3} c$, and the hydrogen ground state hyperfine interval yields a staggering $\mathbf{v} \sim 3.6 \times 10^{8} \mathrm{c}$.

The estimate (4.7) also applies to classical dipole radiation, to the radiant power absorbed by an antenna. For a given frequency, and $m_{\mathrm{t}} c^{2} /(h \nu) \gg 1$,

$$
\frac{P_{\mathrm{tach}}}{P_{\mathrm{ph}}}<\frac{\hat{\beta}}{\hat{\alpha}} \frac{m_{\mathrm{t}} c^{2}}{h \nu}
$$

so that $P_{\mathrm{tach}} / P_{\mathrm{ph}}\left(\nu_{\mathrm{ph}}^{\mathrm{peak}}\right)<4.6 \times 10^{-5}$ at the maximum of the photon energy density at $2.73 \mathrm{~K}$. The chances to detect the cosmic tachyon radiation, i.e., to observe the limit (4.2) instead of the Rayleigh-Jeans law, improve with increasing wavelength, e.g., $P_{\text {tach }} / P_{\text {ph }}(7.3 \mathrm{MHz})<1$. The Planckian shape of the cosmic black-body radiation has not been tested for wavelengths above $50 \mathrm{~cm}, c f$. [12].

As for the thermodynamic variables, their low temperature limits can readily be calculated [36]. The partition function reads

$$
\log Z=-\frac{2 V}{h^{3}} \int_{|\mathbf{p}|>m_{\mathrm{t}} c} \mathrm{~d}^{3} p \log [1-\exp (-\beta h \nu(p))],
$$

and we obtain, for $m_{\mathrm{t}} c^{2} /(k T) \gg 1$,

$$
\begin{aligned}
U & \sim 16 \pi \zeta(3) \frac{m_{\mathrm{t}} k^{3}}{c h^{3}} V T^{3}, \quad N \sim \frac{4}{3} \pi^{3} \frac{m_{\mathrm{t}} k^{2}}{c h^{3}} V T^{2}, \\
P V & =-F \sim \frac{1}{2} U \sim \frac{6 \zeta(3)}{\pi^{2}} N k T, \\
S & \sim \frac{1}{2} c_{V} \sim \frac{3}{2} \frac{U}{T} \sim \frac{18 \zeta(3)}{\pi^{2}} N k,
\end{aligned}
$$

with $\zeta(3) \approx 1.202$. Entropy and the thermal equation of state are, in the indicated leading order of the asymptotic expansion, independent of the tachyon mass, but not so the caloric equation. One readily calculates the ratio of photon and tachyon density as well as the ratio of the corresponding energy densities in the low temperature regime,

$$
\frac{N_{\mathrm{tach}}}{N_{\mathrm{ph}}} \sim \frac{\pi^{2}}{12 \zeta(3)} \frac{m_{\mathrm{t}} c^{2}}{k T}, \quad \frac{U_{\mathrm{tach}}}{U_{\mathrm{ph}}} \sim \frac{30 \zeta(3)}{\pi^{4}} \frac{m_{\mathrm{t}} c^{2}}{k T} .
$$

We find, with a background temperature of $2.73 \mathrm{~K}$, $N_{\mathrm{tach}} / N_{\mathrm{ph}} \approx 6.2 \times 10^{6}$ and $U_{\mathrm{tach}} / U_{\mathrm{ph}} \approx 3.4 \times 10^{6}$, but as we have seen, the high tachyon density cannot compensate the very small ratio of tachyonic and electric fine structure constants, at least not in the microwave regime.

\section{Conclusion}

The cosmology advanced here is at least in two ways deviating from the Einsteinian approach based on the postu- lates of homogeneity and isotropy and the relativity principle. Firstly, the cosmic evolution mentioned in the introduction deforms the open 3-space globally, without changing its constant curvature. This evolution is topologically induced; a multiply connected 3-space is a prerequisite for it. Homogeneity and isotropy are implemented only to the extend that the 3 -space retains its constant curvature. (The curvature radius scales, as in traditional RobertsonWalker cosmology, with the expansion factor.) Locally, the 3 -space still admits a six-dimensional continuous symmetry group, but globally homogeneity and isotropy are destroyed by the multiple connectivity. This is also evident from the qualitative behavior of world-lines, in particular from the emergence of a domain of chaoticity in the open 3-space.

The second difference to Einsteinian as well as Newtonian cosmology is the space-time view itself. Relativity principles asserting the physical equivalence of observations of galactic observers and observers uniformly moving in the galaxy grid are not compatible with the causality principle, if events are connected by superluminal signals [9]. The space concept of Newtonian mechanics is that of the void, generated by rectangular coordinate axes in our imagination. In the theory of special relativity this does not change at all, and even in general relativity this space conception prevails by the possibility to introduce locally geodesic coordinates. The Euclidean metric is replaced by a more complicated one, but space remains a geometric construct of our imagination, the void labeled by coordinates and quantified by a metric, also see $[1$, 41] for discussions on the reality of the space expansion. In this context relativity principles asserting the equivalence of observations made by different observers uniformly moving in the void are indeed natural. However, a purely geometric space conception and the relativity principles associated with it are in my opinion inappropriate in cosmology. Cosmic space is generated by the galaxy grid, which provides a natural reference frame, practically realized by the Planckian microwave background. The consequences of this frame have never been seriously faced, though it is widely acknowledged that comoving coordinates define a distinguished cosmic time. The state of rest can be defined with respect to the galaxy background, and uniform motion and rest become easily distinguishable states. Whether an observer is at rest or in uniform motion with respect to the microwave background, this can really be unambiguously decided, quantitatively, by measuring the dipole anisotropy of the background temperature, caused by a Doppler shift. If tachyons are defined with respect to this comoving frame, as we have done in this paper, a causality problem does not even arise, since the time order of events is unambiguously defined by this frame. All uniformly moving observers can relate their proper time to cosmic time, by determining their motion relative to the background radiation, and can arrive so at the same conclusions on cause and effect. To figure out the causality of an experiment involving tachyons, one has to connect the lab to the rest of the universe and to determine its motion relative to the galaxy background. This is quite 
possible today, thanks to the microwave radiation; the solar barycenter is moving with $370 \mathrm{~km} / \mathrm{s}$, cf. [12], fast enough to even neglect the relative motions of the Earth in a first approximation [57].

In this paper a classical field theory for tachyons is studied, complemented by a semiclassical attachment of frequency to the tachyonic rays. The assumed validity of the de Broglie relation for tachyons is supported by the fact that the classical action appears as the phase of the freely propagating spectral waves. The most promising approach to second quantization outside the light cone is still that of Feinberg $[27,29]$, based on an incomplete set of eigenmodes (and a scalar field theory); the truncation of the momentum integration in the partition function (4.9) is in fact borrowed from [27]. Feinberg's controversial noninvariant vacuum is not an obstacle in a cosmological setting, if one defines it with respect to the comoving frame, and then his quantization is in my opinion consistent, at least as far as the free field is concerned. (Locally, the cosmic reference frame as defined by the galaxy grid is manifest by a permeability tensor $[40,41]$.) However, before one starts to endeavor on a systematic quantization procedure of the Proca field with negative mass square, one needs a good command of the classical theory, of wave propagation and generation outside the light cone, and of interactions with subluminal currents. One would also have to think over what is to be explained by tachyonic quantum effects. If one contemplates on tachyons, one has to decide where to search for them, where could a tachyon field manifest? Does it affect planetary perihelion shifts, or nuclear interactions? The best chances today to find evidence for a tachyon field are presumably high-precision experiments in atomic physics, and that is the reason to consider the tachyon potential as a perturbation of the Coulomb potential. The coupling strength of the tachyon potential is by eleven orders of magnitude smaller than the coupling strength of the Coulomb potential; the classical tachyon field gives only a very tiny contribution to the Lamb shift, a small fraction of the QED contribution, and tachyonic quantum fluctuations may be much harder to detect than semiclassical corrections to energy levels. Rydberg transitions give rise to very speedy tachyons; transitions induced by tachyon radiation as well as the tachyonic analog to the photoelectric effect in Rydberg atoms will be discussed elsewhere.

The author acknowledges the support of the Japan Society for the Promotion of Science. I would like to thank C.V. Vishveshwara for initiating my visit to the Indian Institute of Astrophysics, Bangalore. The hospitality and stimulating atmosphere of the Centre for Nonlinear Dynamics, Bharathidasan University, Trichy, the Institute of Mathematical Sciences, Madras, and the Tata Institute of Fundamental Research, Bombay, are likewise gratefully acknowledged. I'd like to thank Nandor Balazs and George Contopoulos for extensive discussions on causality, and Svend Rugh for criticism and numerous comments on an earlier version of the typescript. I also thank Gordon Drake for communicating me the revised $\mathrm{He}^{+}$Lamb shift prior to publication. The calculations for Figure 1 were carried out in the Service de Physique Théorique de
Saclay, I once more thank Daniel Bessis for arranging my stay there.

\section{Appendix A: Ground state eigenfunction in the tachyon potential}

We calculate the ground state eigenfunction of $(3.1,3.2)$ in linear order in $\lambda:=\beta / \alpha$. The setting is as in (3.1-3.5). We use a perturbative method frequently applied to ground states [58], which is much simpler than Schrödinger perturbation theory, as it does not involve eigenfunction expansions. In (3.2) we substitute $E=E_{0}+\lambda E_{1}+O\left(\lambda^{2}\right)$ and $u_{l, E}(r)=u_{0}(r)+\lambda u_{1}(r)+O\left(\lambda^{2}\right)$. A formal power series expansion of (3.2) in $\lambda$ gives for the zeroth and first order

$$
\begin{gathered}
\left(-\mathrm{d}^{2} / \mathrm{d} r^{2}+h(r ; n, l)\right) u_{0}=0, \\
h(r ; n, l):=\frac{1}{a^{2} n^{2}}-\frac{2}{a r}+\frac{l(l+1)}{r^{2}}, \\
\left(-\mathrm{d}^{2} / \mathrm{d} r^{2}+h(r ; n, l)\right) u_{1}=\frac{2}{a}\left(\frac{E_{1}}{\alpha}-\frac{1}{r} \cos (\mu r)\right) u_{0},
\end{gathered}
$$

respectively. We define a scalar product $\langle u, v\rangle:=$ $\int_{0}^{\infty} u(r) \bar{v}(r) \mathrm{d} r$, and consider solutions of (A.1, A.2) regular at $r=0, u(0)=0$, and exponentially decaying for large $r$. By applying partial integration twice, we find

$$
\left\langle u_{0},\left(-\mathrm{d}^{2} / \mathrm{d} r^{2}+h(r ; n, l)\right) u_{1}\right\rangle=0 .
$$

In the following we focus on the ground state, $n=1$, $l=0$. The normalized solution $u_{0}$ of (A.1) is given in (3.5). The first order correction $E_{1}$ to the ground state energy, as given in $(3.3,3.5)$, readily follows from (A.2, A.3). Equation (A.2) may thus be written as

$$
\begin{aligned}
& u_{1}^{\prime \prime}(r)-\left(\frac{1}{a^{2}}-\frac{2}{a r}\right) u_{1}(r)=g(r), \\
& g(r):=\frac{4 \mathrm{e}^{-r / a}}{a^{5 / 2}}\left(\cos (\mu r)-\frac{\left(1-a^{2} \mu^{2} / 4\right)}{\left(1+a^{2} \mu^{2} / 4\right)^{2}} \frac{r}{a}\right) .
\end{aligned}
$$

$u_{0}$ in (3.5) is also a solution of the homogeneous equation in (A.4). A second fundamental solution is $\varphi_{0}=-\mathrm{e}^{r / a}+$ $2 a^{-1} r \mathrm{e}^{-r / a} \operatorname{Ei}(2 r / a)$, which diverges exponentially. Here, $\operatorname{Ei}(2 r / a)$ is just meant as an antiderivative of $r^{-1} \mathrm{e}^{2 r / a}$. The Wronskian is $u_{0} \varphi_{0}^{\prime}-u_{0}^{\prime} \varphi_{0}=2 a^{-3 / 2}$, and $u_{1}=$ $\left(a^{3 / 2} / 2\right)\left(\varphi_{0} \int u_{0} g \mathrm{~d} r-u_{0} \int \varphi_{0} g \mathrm{~d} r\right)$ solves (A.4). Integrations over $\operatorname{Ei}(2 r / a)$ can be avoided by partial integration, and the integration constants can be chosen in a way that $\operatorname{Ei}(2 r / a)$ drops out; the remaining integrations are 
elementary. A solution of (A.4) decaying at infinity is

$$
\begin{aligned}
u_{1}(r)= & \frac{2 \mathrm{e}^{-r / a}}{a^{1 / 2}\left(1+a^{2} \mu^{2} / 4\right)^{2}}\left[( 1 - a ^ { 2 } \mu ^ { 2 } / 4 ) \left(\frac{r^{2}}{a^{2}}-\frac{r}{a}\right.\right. \\
& \left.+\frac{r}{a} \log (\mu r)-\frac{1}{2}+\frac{1}{2} \cos (\mu r)-\frac{r}{a} \operatorname{ci}(\mu r)\right) \\
& \left.-\mu a\left(\frac{1}{2} \sin (\mu r)-\frac{r}{a} \operatorname{si}(\mu r)\right)\right], \\
\operatorname{si}(\mu r):= & -\frac{\pi}{2}+\int_{0}^{r} \frac{\sin (\mu r)}{r} \mathrm{~d} r, \\
\operatorname{ci}(\mu r):= & \gamma+\log (\mu r)+\int_{0}^{r} \frac{\cos (\mu r)-1}{r} \mathrm{~d} r \\
u_{1}(r \rightarrow 0)= & \frac{-2 r}{a^{3 / 2}\left(1+a^{2} \mu^{2} / 4\right)^{2}}\left(\left(1-a^{2} \mu^{2} / 4\right)(1+\gamma)\right. \\
& \left.+\frac{\pi}{2} a \mu+\frac{1}{2} a^{2} \mu^{2}\right)+O\left(r^{2}\right) .
\end{aligned}
$$

We may still add a constant multiple of $u_{0}$ to $u_{1}$, and choose

$$
\hat{u}_{1}(r):=u_{1}(r)-\left\langle u_{1}, u_{0}\right\rangle u_{0}(r),
$$

so that $\left\langle\hat{u}_{1}, u_{0}\right\rangle=0$. Clearly, $\hat{u}_{1}$ is the only square integrable solution of (A.4) orthogonal to $u_{0}$. The scalar product of $u_{1}$ (defined by (A.5)) and $u_{0}$ is

$$
\begin{array}{r}
\left\langle u_{1}, u_{0}\right\rangle=\frac{1}{\left(1+a^{2} \mu^{2} / 4\right)^{2}}\left[-1+\left(1-a^{2} \mu^{2} / 4\right)\right. \\
\quad \times\left(\frac{3}{2}-\gamma+\frac{1}{2} \log \left(1+a^{2} \mu^{2} / 4\right)\right) \\
\left.\quad-a \mu \arctan \frac{2}{a \mu}+\frac{a^{2} \mu^{2}}{1+a^{2} \mu^{2} / 4}\right],
\end{array}
$$

$0<\arctan <\pi / 2 ;$ the integrals needed to calculate (A.8) are all standard. We obtain, for $r \rightarrow 0$ and with $\hat{\mu}:=a \mu$, $c f$. (3.8),

$$
\begin{aligned}
\frac{\hat{u}_{1}(r)}{r}= & -\frac{1}{a^{3 / 2}\left(1+\hat{\mu}^{2} / 4\right)^{2}}\left(3+\left(1-\hat{\mu}^{2} / 4\right) \log \left(1+\hat{\mu}^{2} / 4\right)\right. \\
& \left.+2 \hat{\mu} \arctan \frac{\hat{\mu}}{2}-\frac{1}{4} \hat{\mu}^{2}+\frac{2 \hat{\mu}^{2}}{1+\hat{\mu}^{2} / 4}\right)+O(r) \\
= & -\frac{3}{a^{3 / 2}}\left(1+\frac{1}{2} \hat{\mu}^{2}+O\left(\hat{\mu}^{4}\right)\right)+O(r) .
\end{aligned}
$$

The ground state wave function of (3.1) can be assembled as

$$
\Psi(r)=\frac{1}{2 \sqrt{\pi}} \frac{u_{0}(r)}{r}\left(1+\lambda \frac{\hat{u}_{1}(r)}{u_{0}(r)}+O\left(\lambda^{2}\right)\right) .
$$

Because of $\left\langle\hat{u}_{1}, u_{0}\right\rangle=0$, we find $\int_{\mathrm{R}^{3}}|\Psi(r)|^{2} \mathrm{~d} \mathbf{x}=1+O\left(\lambda^{2}\right)$ the wave function is normalized in linear order. For the calculation of the ground state hyperfine interval in Section 3, $c f .(3.26)$, we need

$$
|\Psi(0)|^{2}=\frac{1}{\pi a^{3}}\left(1+\left.\lambda a^{3 / 2} \frac{\hat{u}_{1}(r)}{r}\right|_{r=0}+O\left(\lambda^{2}\right)\right),
$$

with $\hat{u}_{1}(r) / r$ as in (A.9).

\section{References}

1. A. Sandage, Ann. Rev. Astron. Astrophys. 26, 561 (1988).

2. N.A. Bahcall et al., Science 284, 1481 (1999).

3. F.J. Dyson, Rev. Mod. Phys. 51, 447 (1979).

4. J.A. Wheeler, in The Physicist's Conception of Nature, edited by J. Mehra (Reidel, Dordrecht, 1973).

5. R. Tomaschitz, J. Math. Phys. 32, 2571 (1991).

6. R. Bowen, J. Diff. Eq. 18, 333 (1975).

7. D. Sullivan, Publ. Math. I.H.E.S. 50, 171 (1979).

8. N.L. Balazs, A. Voros, Phys. Rep. 143, 109 (1986).

9. R. Tomaschitz, Chaos, Solitons \& Fractals 7, 753 (1996).

10. R. Tomaschitz, Complex Systems 6, 137 (1992).

11. R. Tomaschitz, Physica D 34, 42 (1989).

12. G.F. Smoot, D. Scott, in C. Caso et al., Eur. Phys. J. C 3, 1 (1998).

13. R. Tomaschitz, J. Math. Phys. 34, 3133 (1993).

14. R. Tomaschitz, in Chaotic Dynamics: Theory and Practice, edited by T. Bountis (Plenum, New York, 1992).

15. Y. Colin de Verdière, Astérisque 132, 259 (1985).

16. H. Poincaré, Papers on Fuchsian Functions (Springer, New York, 1985).

17. L. Nottale, Fractal Space-Time and Micro Physics (World Scientific, Singapore, 1993).

18. M.S. El Naschie, in Quantum Mechanics, Diffusion, and Chaotic Fractals, edited by M.S. El Naschie et al. (Elsevier, Oxford, 1995).

19. M.S. El Naschie, Chaos, Solitons \& Fractals 8, 753 (1997); ibid. 9, 1445 (1998).

20. R. Tomaschitz, J. Math. Phys. 35, 1573 (1994).

21. S.E. Rugh, in Deterministic Chaos in General Relativity, edited by D. Hobill et al. (Plenum, New York, 1994).

22. S.E. Rugh, in Quantum Mechanics, Diffusion, and Chaotic Fractals, edited by M.S. El Naschie et al. (Elsevier, Oxford, 1995).

23. G. Contopoulos, Proc. Roy. Soc. A 431, 183 (1990); ibid. A 435, 551 (1991).

24. G. Contopoulos, in Deterministic Chaos in General Relativity, edited by D. Hobill et al. (Plenum, New York, 1994).

25. S. Tanaka, Progr. Theoret. Phys. 24, 171 (1960).

26. Ya.P. Terletsky, Sov. Phys. Dokl. 5, 782 (1961).

27. G. Feinberg, Phys. Rev. 159, 1089 (1967).

28. G. Feinberg, Sci. Am. 222, 69 (1970).

29. G. Feinberg, Phys. Rev. D 17, 1651 (1978).

30. R. Newton, Science 167, 1569 (1970).

31. P.C.W. Davies, Nuovo Cimento B 25, 571 (1975).

32. E. Chaliasos, Physica A 144, 390 (1987).

33. R. Tomaschitz, Chaos, Solitons \& Fractals 8, 761 (1997).

34. R. Tomaschitz, Intern. J. Mod. Phys. D 7, 279 (1998).

35. R. Tomaschitz, Class. Quant. Grav. 16, 3349 (1999).

36. R. Tomaschitz, Int. J. Mod. Phys. A 14, 4275 (1999); ibid. A 14, 5137 (1999); ibid. A 15, 3019 (2000).

37. K. Matsuzaki, M. Taniguchi, Hyperbolic Manifolds and Kleinian Groups (Clarendon, Oxford, 1998).

38. P.A.M. Dirac, in The Physicist's Conception of Nature, edited by J. Mehra (Reidel, Dordrecht, 1973).

39. F.J. Dyson, in Aspects of Quantum Theory, edited by A. Salam, E.P. Wigner (Cambridge Univ. Press, 1972).

40. R. Tomaschitz, Chaos, Solitons \& Fractals 9, 1199 (1998). 
41. R. Tomaschitz, Astrophys. Space Sci. 259, 255 (1998); ibid. 271, 181 (2000).

42. J. Mould, R.C. Kennicutt, W. Freedman, Rep. Prog. Phys. 63, 763 (2000).

43. J.A. Wheeler, R.P. Feynman, Rev. Mod. Phys. 17, 157 (1945); ibid. 21, 425 (1949).

44. A.S. Goldhaber, M.M. Nieto, Rev. Mod. Phys. 43, 277 (1971).

45. Th. Udem et al., Phys. Rev. Lett. 79, 2646 (1997).

46. P.J. Mohr, B.N. Taylor, J. Phys. Chem. Ref. Data 28, 1713 (1999).

47. E.W. Hagley, F.M. Pipkin, Phys. Rev. Lett. 72, 1172 (1993).

48. U. Jentschura, K. Pachucki, Phys. Rev. A 54, 1853 (1996).
49. S. Bourzeix et al., Phys. Rev. Lett. 76, 384 (1996).

50. D.J. Berkeland, E.A. Hinds, M.G. Boshier, Phys. Rev. Lett. 75, 2470 (1995).

51. M. Weitz et al., Phys. Rev. A 52, 2664 (1995).

52. V. Meyer et al., Phys. Rev. Lett. 84, 1136 (2000).

53. J.R. Sapirstein, D.R. Yennie, in Quantum Electrodynam$i c s$, edited by T. Kinoshita (World Scientific, Singapore, 1990).

54. A. van Wijngaarden, F. Holuj, G.W.F. Drake, Phys. Rev. A (to appear).

55. P J. Mohr et al., Phys. Rep. 293, 227 (1998).

56. W. Liu et al., Phys. Rev. Lett. 82, 711 (1999).

57. R. Tomaschitz, Celest. Mech. Dyn. Astron. (to appear).

58. A. Dalgarno, J.T. Lewis, Proc. Roy. Soc A 233, 70 (1955). 